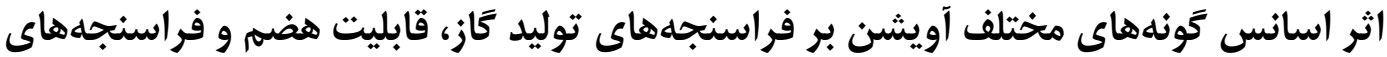

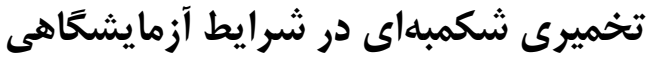

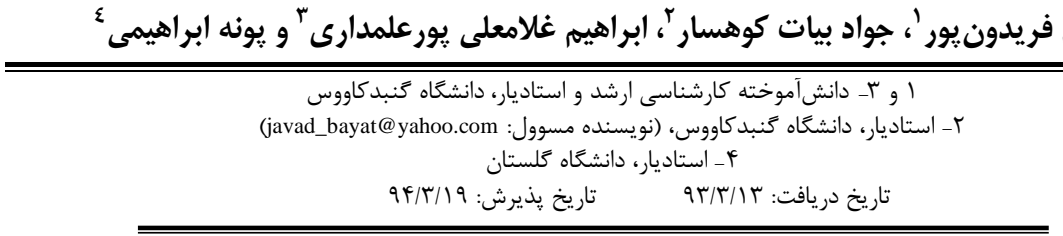

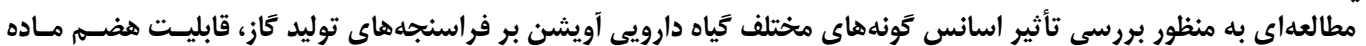

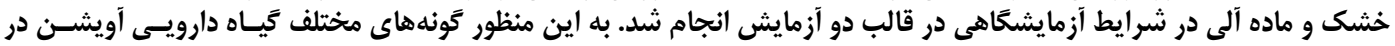

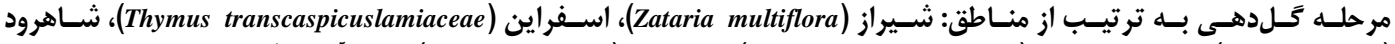
(Thymus fallax)

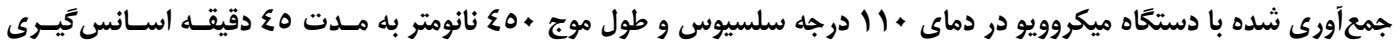

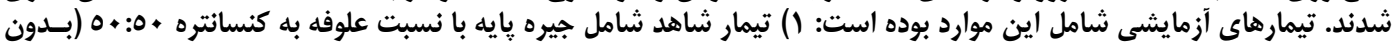

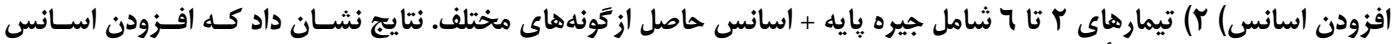

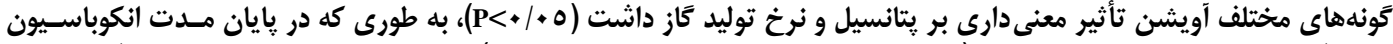

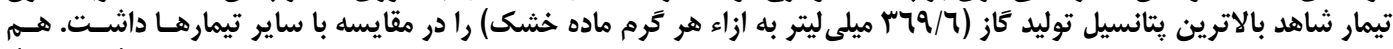

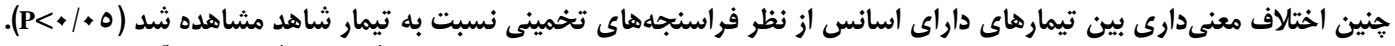

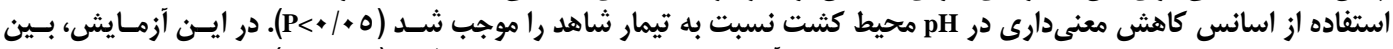

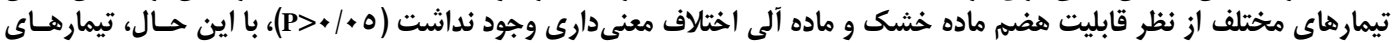

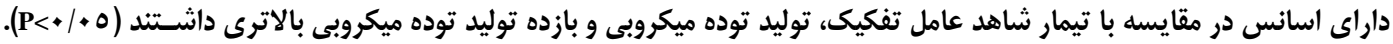

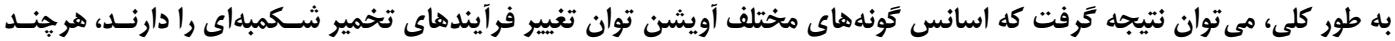

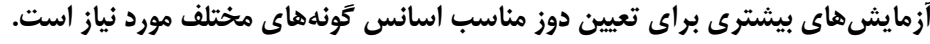

وازههاى كليدى: اسانس، تخمير شكمبه، قابليت هضم، شرايط آزمايشكاهى

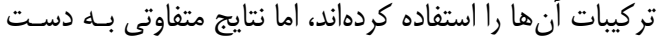

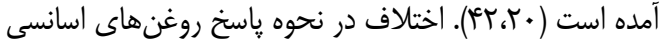

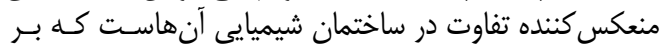

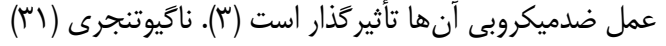

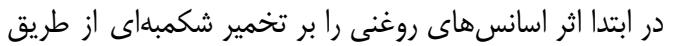

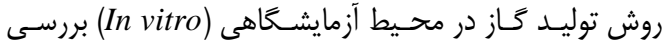

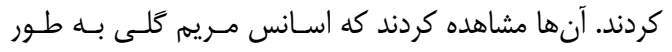

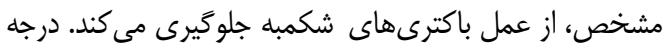

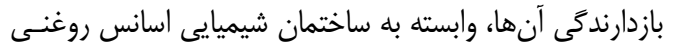
مورد استفاده است.

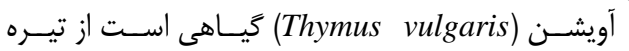
نعناعيان (lamiaceae) كه در نواحى مختلف مديترانه و برخى (T) إنى

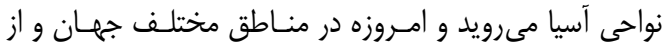

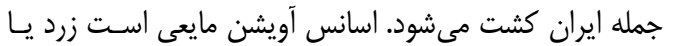

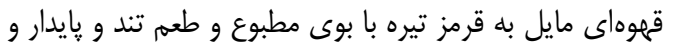

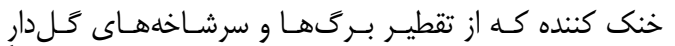

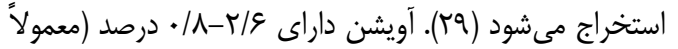

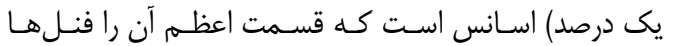

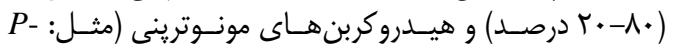

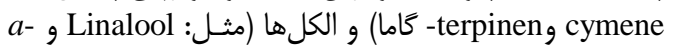

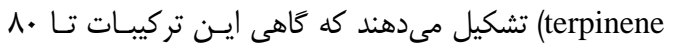

متخصصين تغذيه نشخوار كنندكان، بــه دنبـال اسـتفاده از

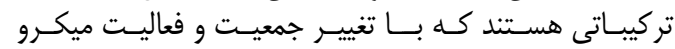

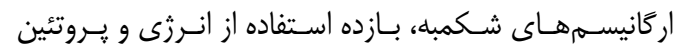

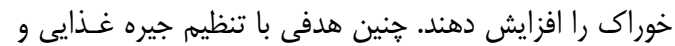

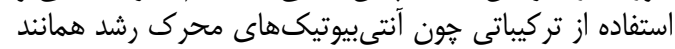

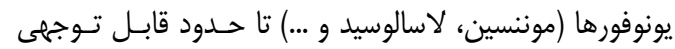

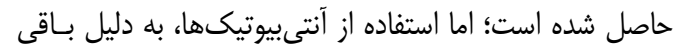

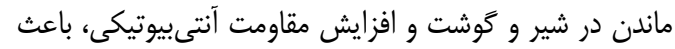

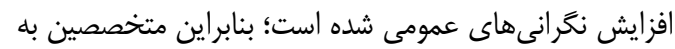

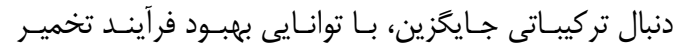

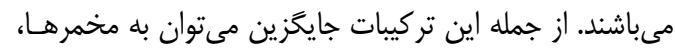

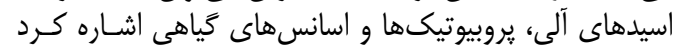

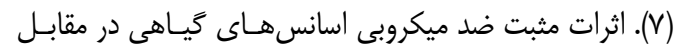

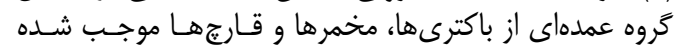

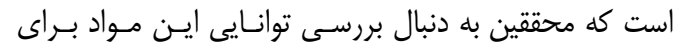

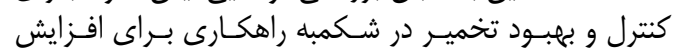

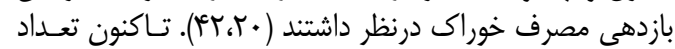

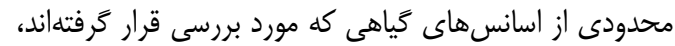

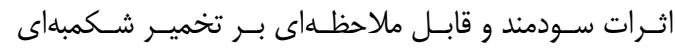

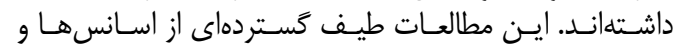


انجام شد. تيمارهاى آزمايشى عبارت بودند از: () تيمار شـاهد

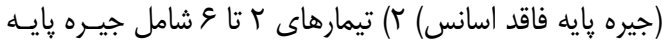

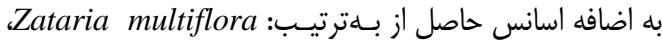
،Thymus fallax ،lamiaceae Thymus transcaspicus .Thymus vulgaris , Thymus transcaucasicus جيره يايه بر اساس ماده خشك شامل: علوفه (يونجــه) +

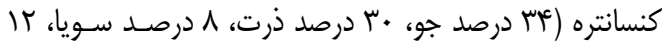

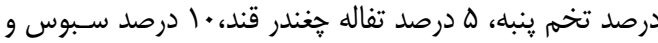

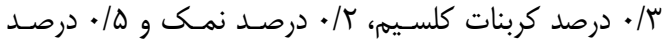

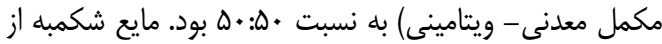

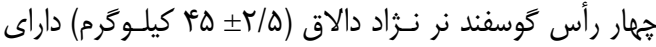

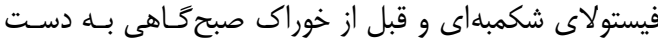

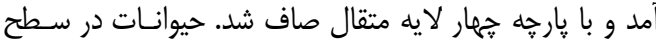

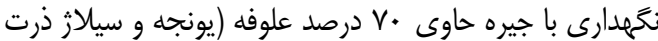

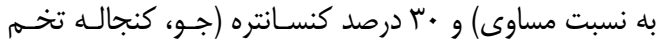

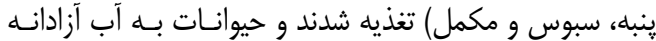

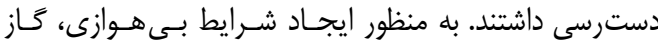
دى اكسيد كربن به مايع شكمبه صاف شده تزريق شد و در در بـ بن

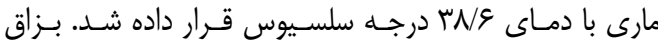

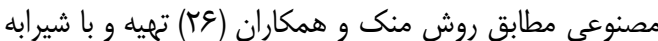

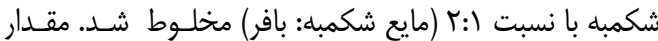

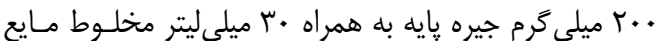

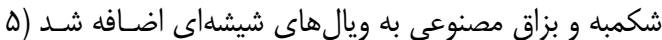

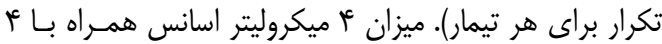

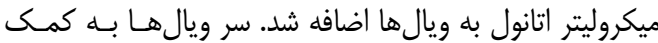

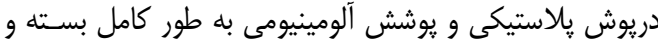

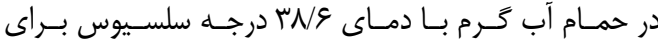

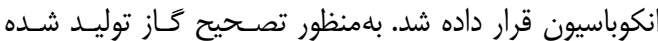

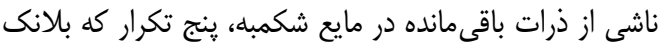

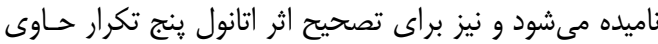

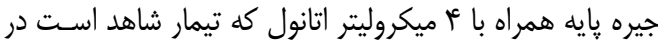

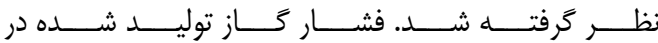

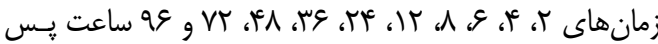

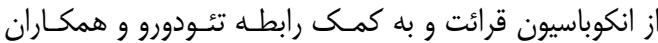

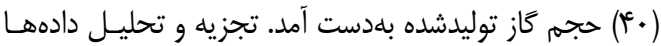

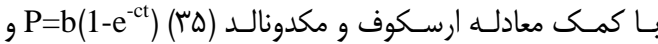

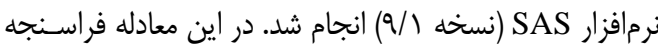

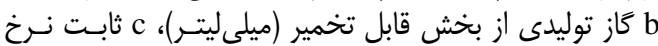

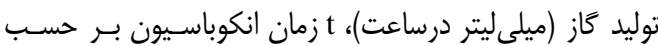

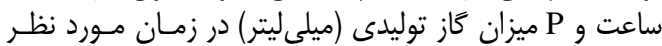

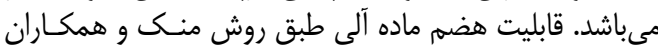

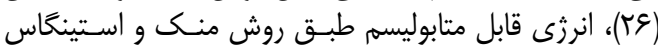

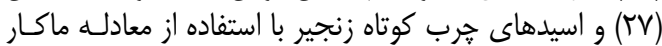

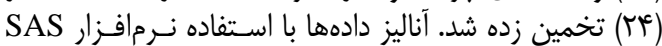

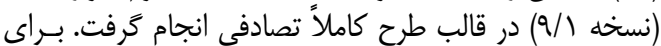

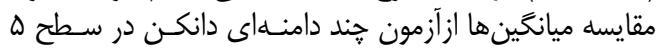
درصد خطا استفاده شد.

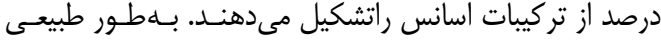

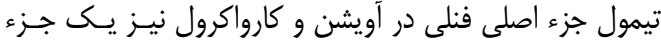
فرعى است (T) تيمول.

فعاليت بيولوزيك اسانس استها به اجزاى تشكيل دهنده آنهات آنها

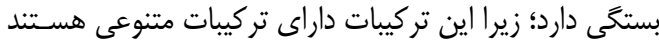

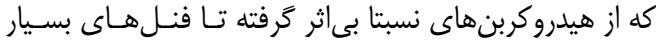

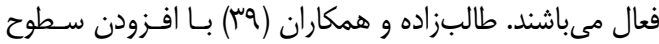

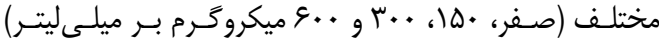

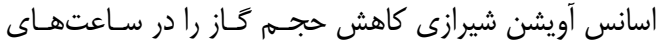

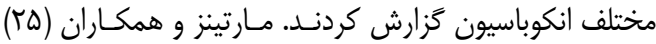

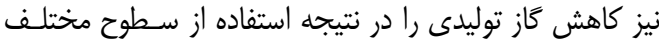

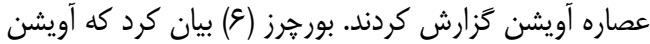

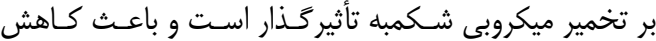

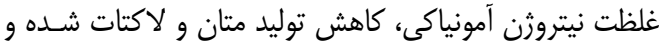
دآميناسيون را محدود مي كند. إندان.

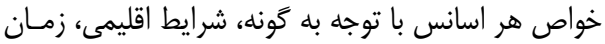

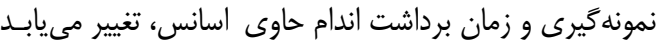

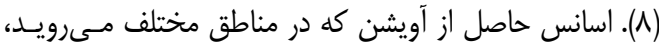

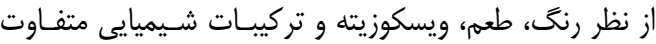

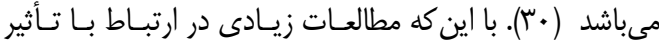

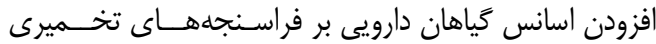

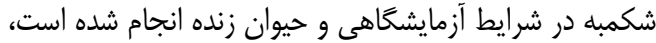

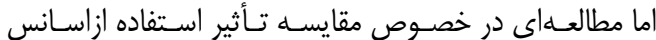

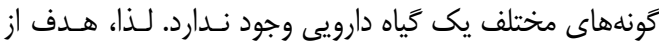

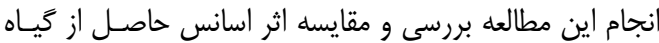

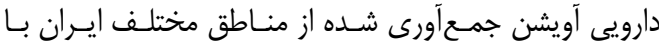

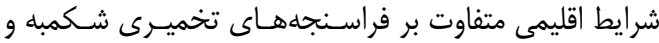

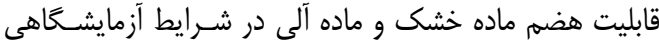

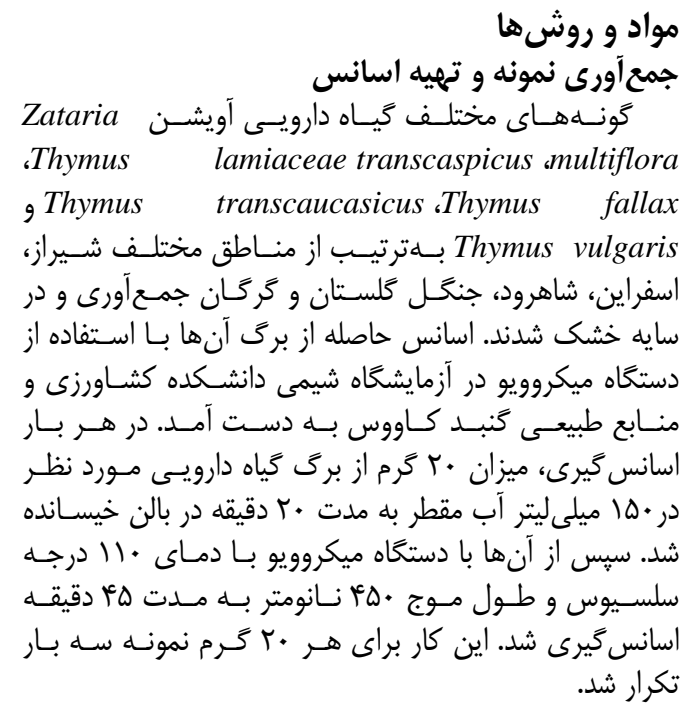

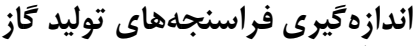

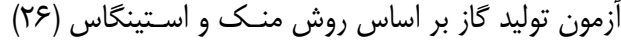


است. نتايج نشان داد كه افزودن اسانس تـأثير معنسى دارى بـر

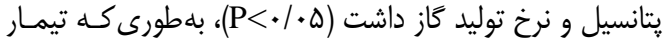

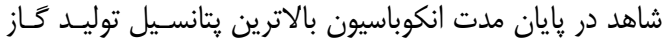

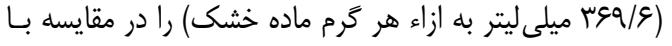

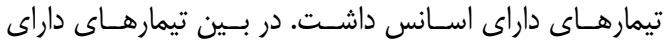

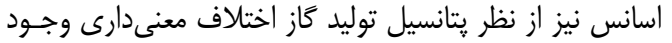

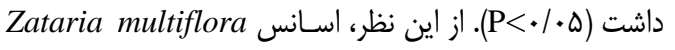

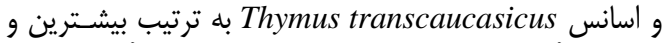

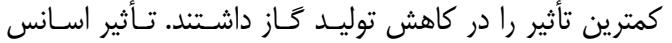

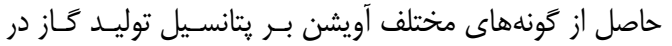

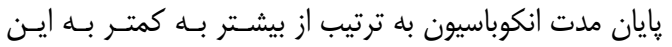

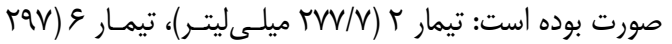

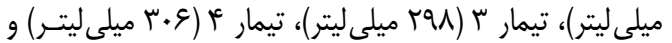

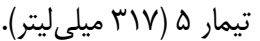

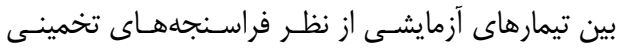

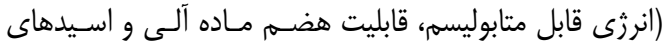

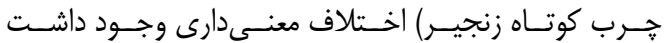

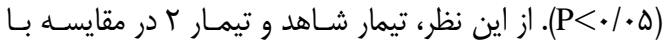

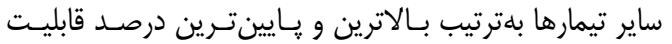

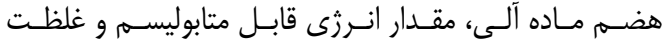

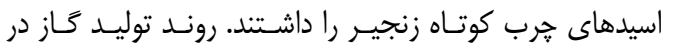

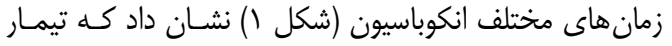

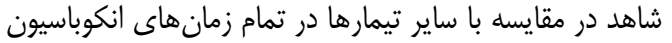

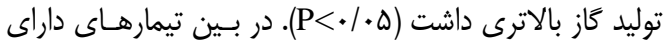

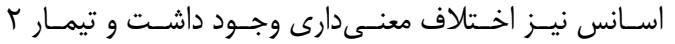

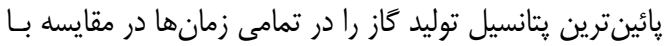
ساير تيمارها داشت.

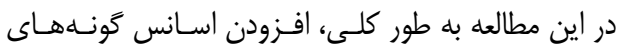

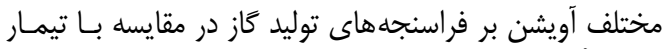

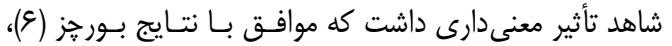

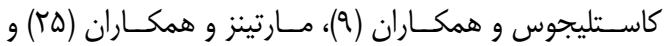

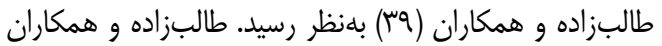

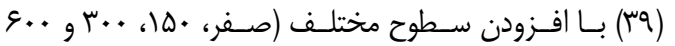

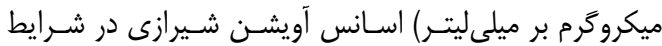

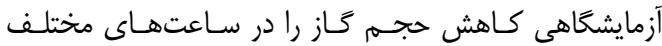

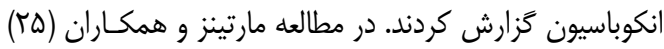

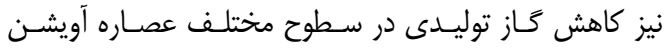

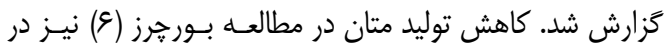

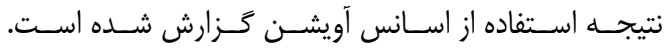

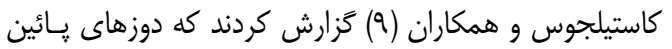

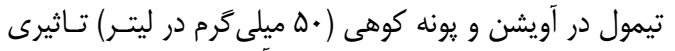

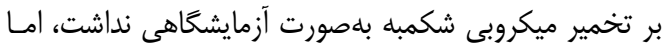

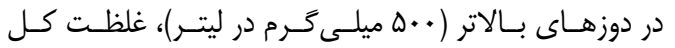

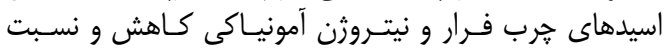

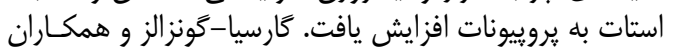
(IV)

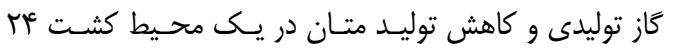

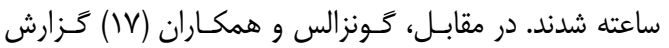
كردند كه اسانسهاى نعنـاع فلفلى، اسـطو خـودوس، نعنـاع،
اندازهتيرى قابليت هضم مـاده خشـك و مــاده آلـى در شرايط كثت بسته

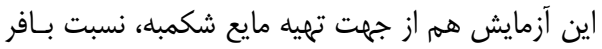

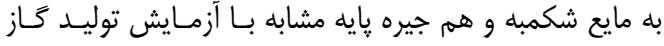

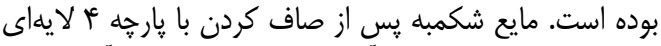

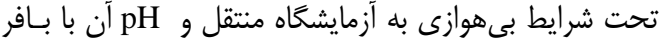

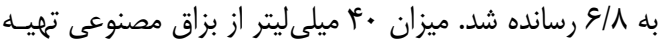

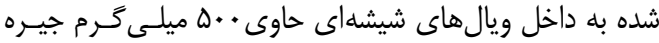

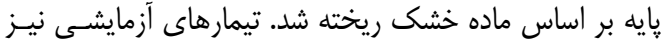

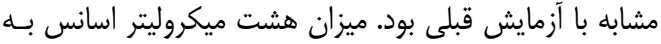

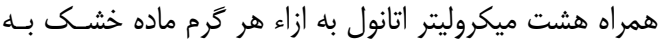

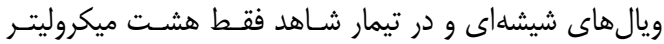

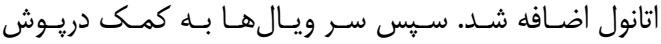

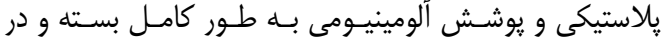

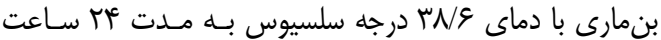

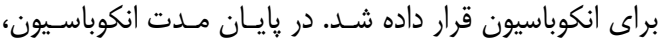

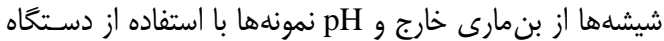

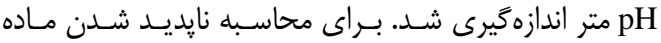

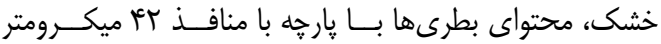

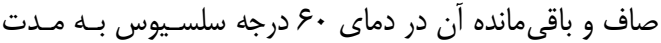

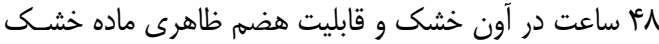

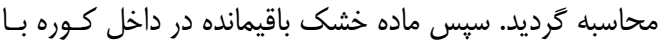

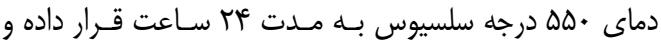

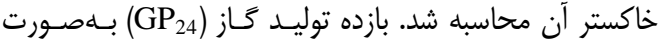

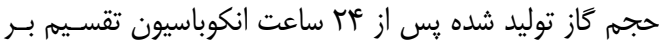

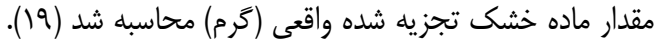

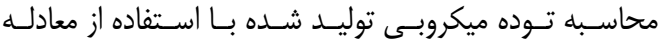

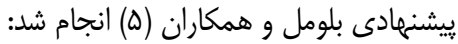

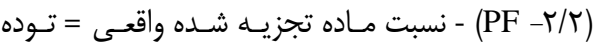

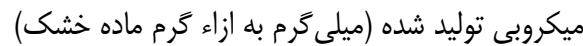

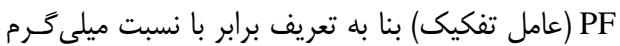

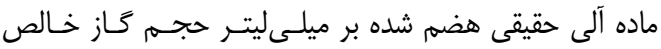

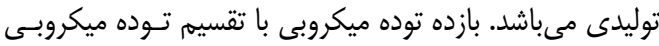

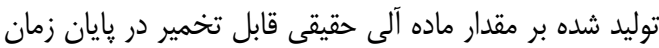

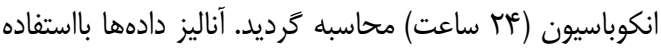

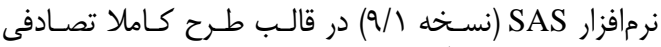

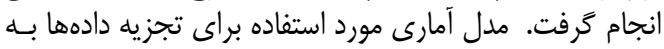

$\mathrm{Y}_{\mathrm{ij}}=+\mathrm{T}_{\mathrm{j}}+\mathrm{e}_{\mathrm{ij}}$

صورت زير بود:

در اين رابطه،

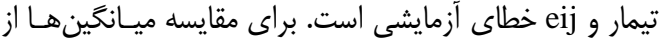
آزمون جند دامنهاى دانكن در سطح ه درصد خطا استفاده شد.

\section{نتايج و بحث

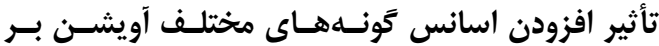 فراسنجههاى توليد كاز أناز}

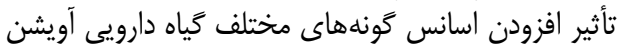

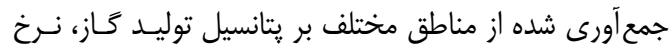

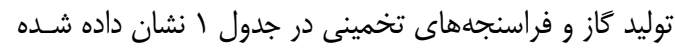




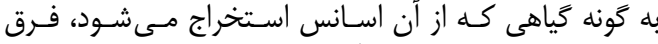

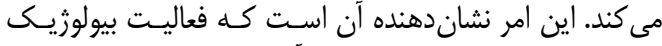

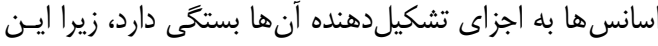

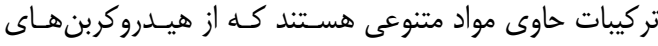

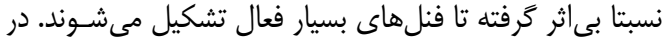

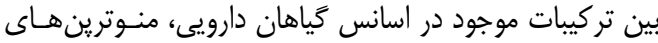

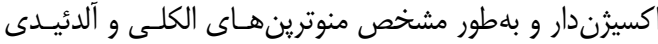

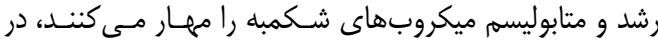

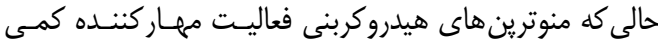

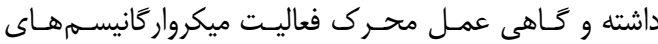
شكمبله را دارا هستند.

خواص هر اسانس بسته به نوع گَونه، شرايط اقليمى محل زئن

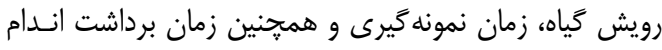

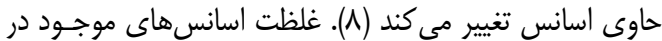

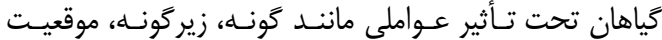

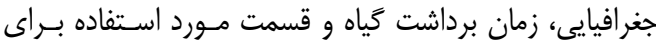

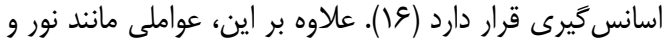

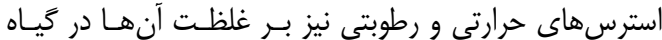

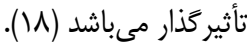

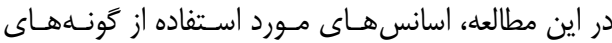

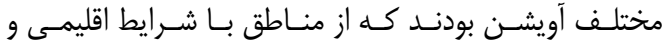

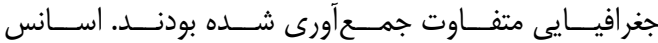

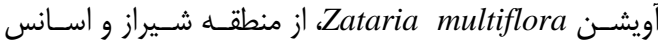
ت transcaucasicus Thymus ترتيب بيشترين و كمترين تأثير را در كاهش توليد كاز داز داشتند.

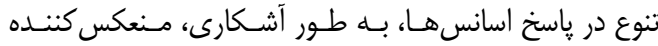

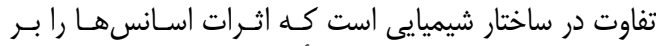

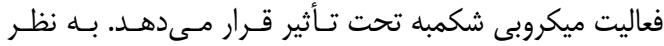

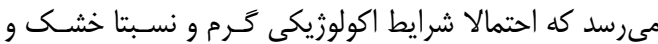

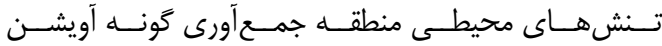
Zataria multiflora

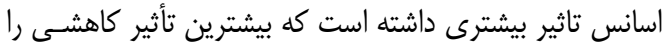
بر توليد كاز داشت.
ريحان، يونه و آويشن (خانواده نعناعيان)، رازيانه، شويد و زيـره

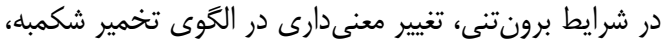

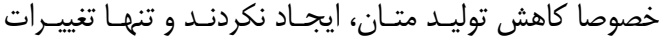

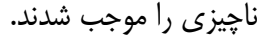

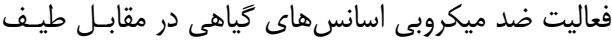

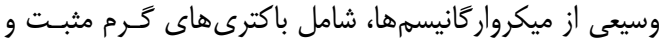

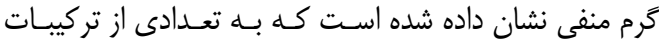

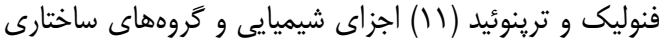

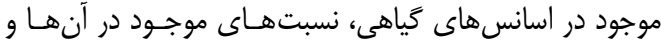

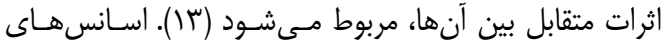

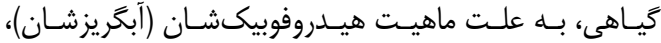

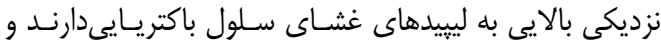

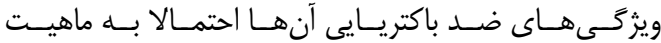

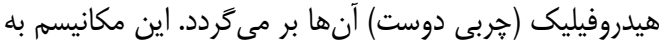

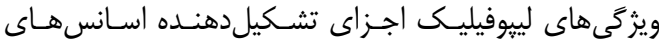

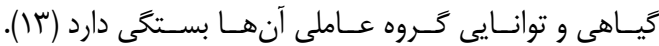

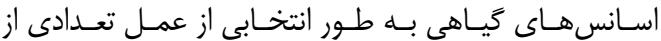

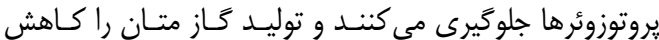

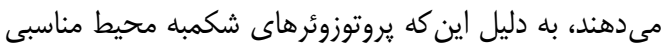

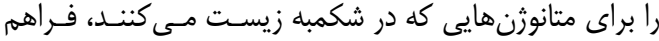

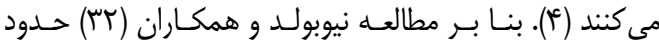

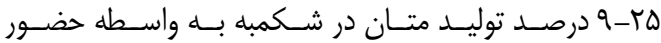
يروتوزوئرهاى مزكىار است.

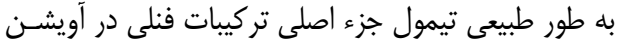

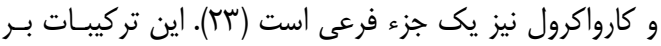

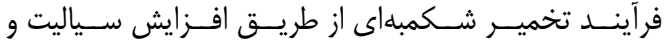

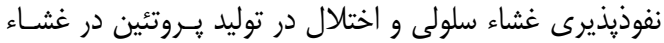

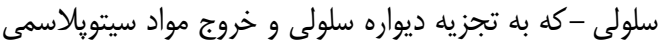

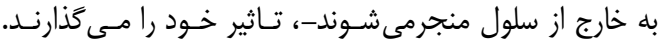

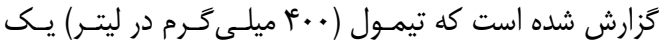

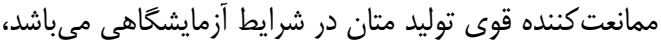

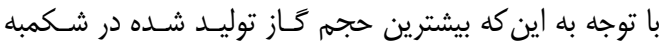

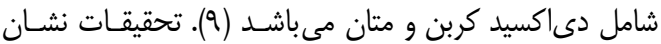

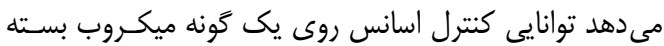

جدول ا- تأثير اسانس گَونهاى مختلف كياه دارويى آويشن بر فراسنجههاى توليد كَاز

Table 1. Effect of various species of Thymus essence on gas production parameters

\begin{tabular}{|c|c|c|c|c|c|}
\hline SCFA & $\mathrm{ME}$ & OMD & C & $(a+b)$ & "تيمارها' \\
\hline $1 / \mu \cdot 9^{a}$ & $1 . / K^{\prime} Y^{\mathrm{a}}$ & $9 \mathrm{~N} \cdot \mathrm{Q}^{\mathrm{a}}$ & $\cdot / \cdot V \Delta T^{D}$ & $r q q / q^{a}$ & 1 \\
\hline.$/ 91 q^{c}$ & $N / \Delta r^{c}$ & $\Delta r / \Delta \Lambda^{c}$ & $.1 .99 r^{\mathrm{c}}$ & $r W / v^{a}$ & r \\
\hline $1 / \mu^{\mathrm{DC}}$ & $N / D S 1^{D C}$ & $\Delta F / Q F^{D C}$ & $.1 .9 \wedge \mathcal{F}^{\mathrm{c}}$ & $r 9 \Lambda / \kappa^{c}$ & r \\
\hline $1 / \cdot \wedge f^{\mathrm{D}}$ & $N / M r^{D}$ & $\Delta q / \cdot \bullet^{0}$ & $\cdot 1 \cdot v \in v^{D}$ & $r \cdot 9 / \mathrm{V}^{\mathrm{c}}$ & f \\
\hline$|/| K^{D}$ & $9 / 1 \cdot 0^{D}$ & $G \cdot \mid \Delta F^{D}$ & $\cdot 1 \cdot V \Delta F^{D}$ & $\mathrm{rIV} / \mathrm{F}^{\mathrm{D}}$ & $\Delta$ \\
\hline $1 / r \cdot 9^{\mathrm{a}}$ & N/VQY ${ }^{D}$ & $\Delta N / F V^{D C}$ & $.1 . \mathrm{vqv}^{\mathrm{a}}$ & $r q v / l^{\circ}$ & 9 \\
\hline $.1 \cdot v q$ & •/FAS & $r / r)$ & r ../. & $r / q V$ & SEM \\
\hline
\end{tabular}

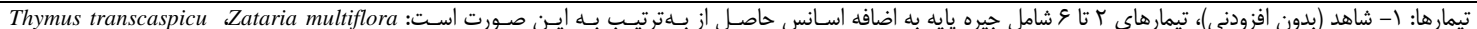

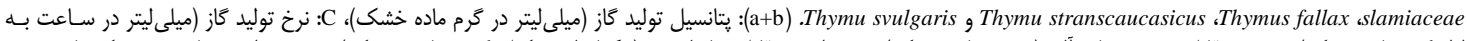

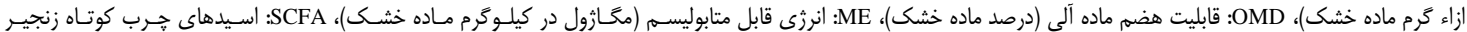

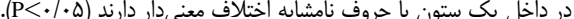




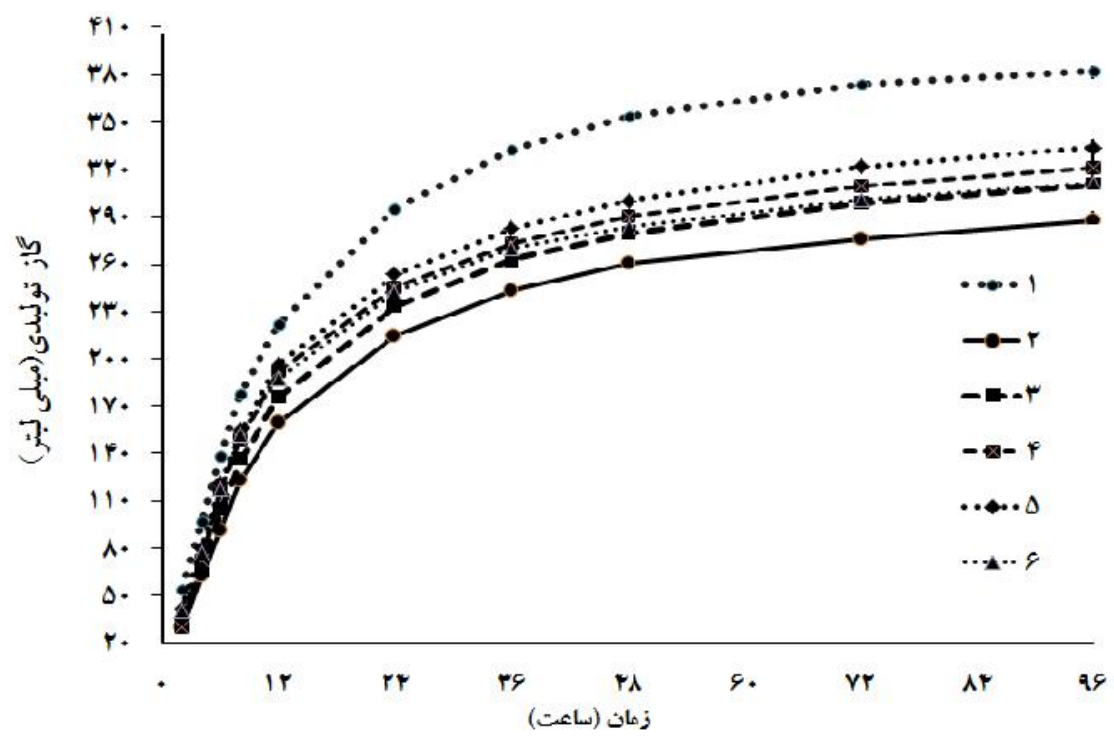

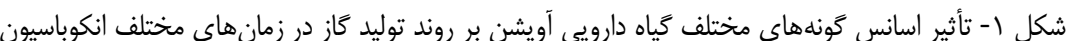

Figure 1. Effect of various species of Thymus essence on gas production curves at different times of incubation

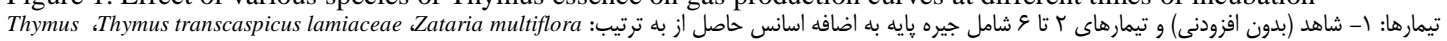

.Thymus vulgaris و Thymus transcaucasicus ,fallax

يروييونات، بوتيرات و آمونياك محيط كشت تأثيرى نداشتند،

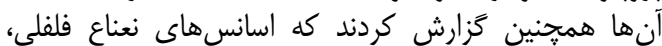

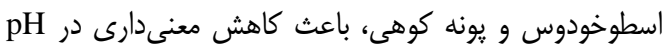

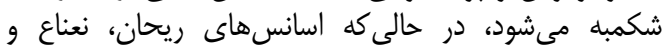

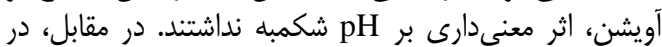

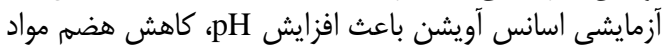

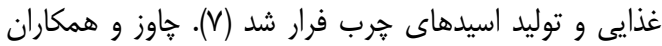

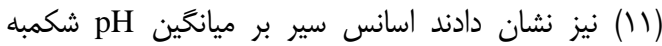

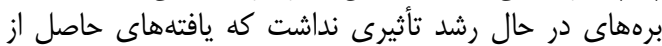

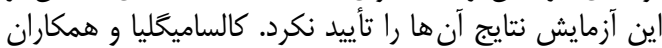

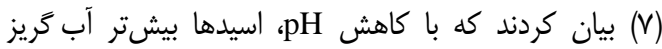

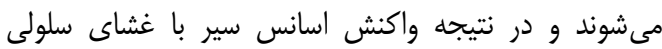

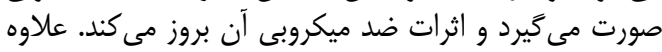

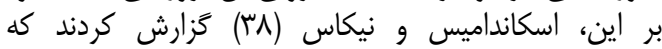

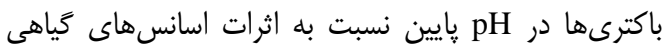

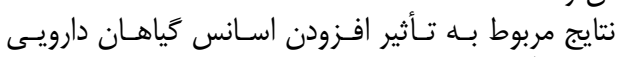

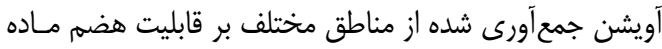

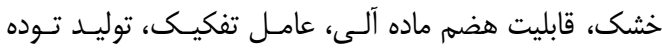

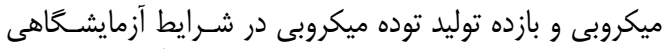

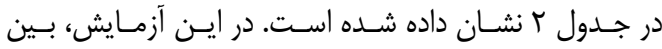

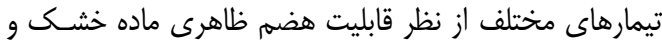

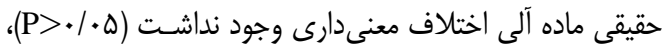

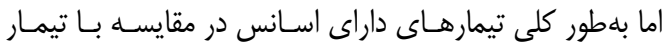

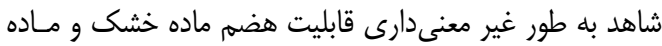

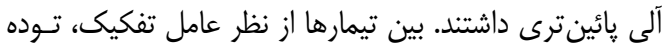

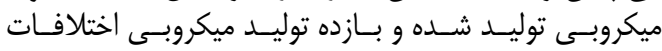

تأثير اسانس تونههاى مختلف آويشن بر قابليت هضم و

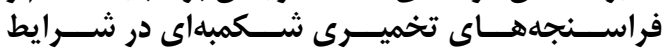

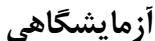

تأثير افزودن اسانس كونهاهاى مختلف كياه دارويى آويشن

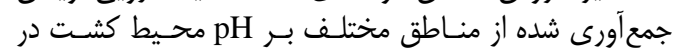

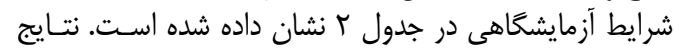

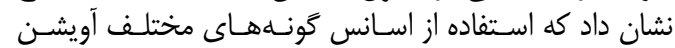

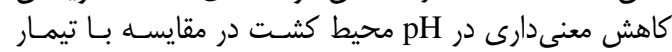

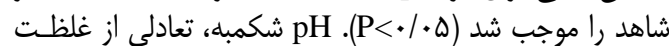

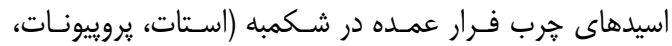

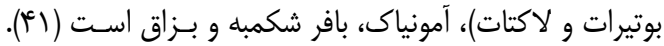

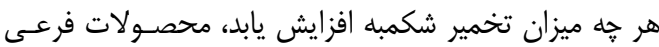

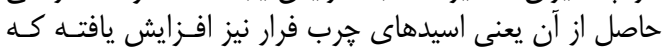

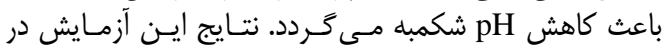

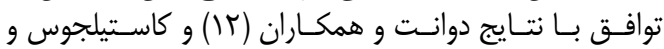

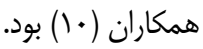

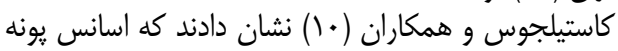

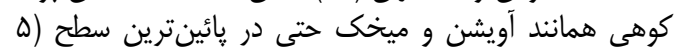

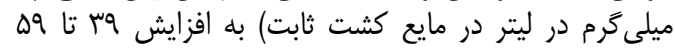

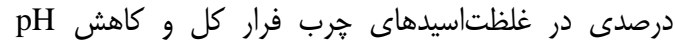

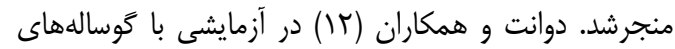

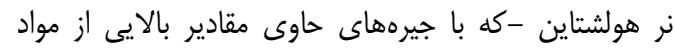

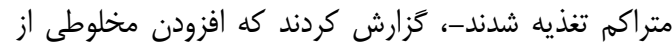

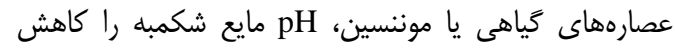

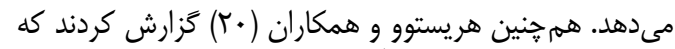

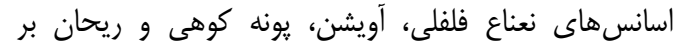

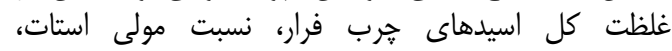


سوبستراى تجزيه شده به صورت حقيقى بر حسب ميلـى گَرم

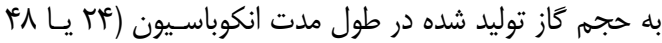

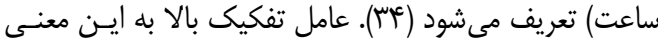

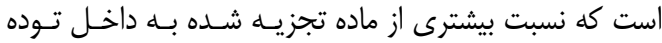

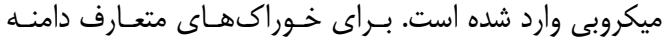

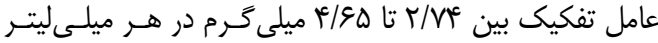

كزارش شده است (أ).

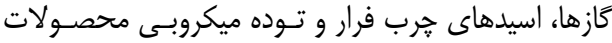

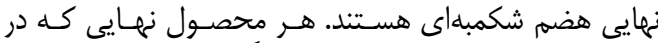

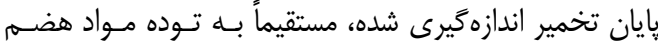

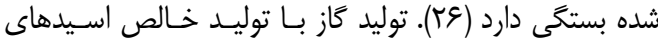

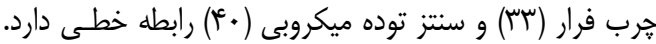

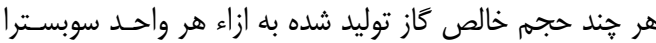

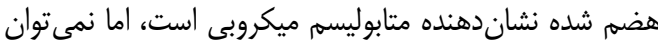

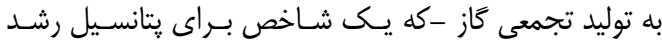

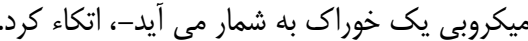

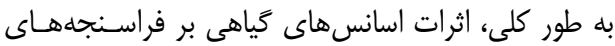

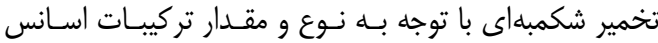

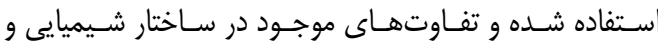

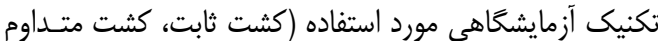

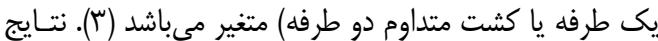

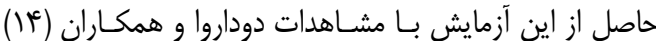

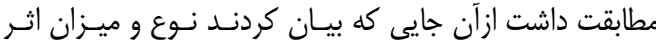

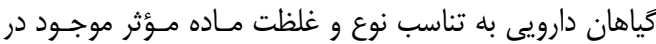

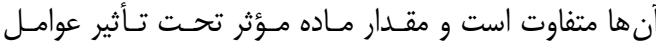

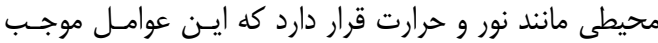

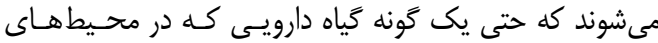
متفاوت رشد كرده داراى مقادير متفاوتى از مواد مؤن مؤثر باشيد.

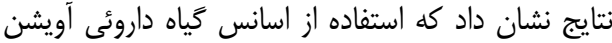

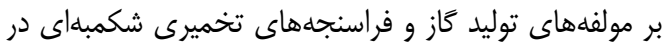

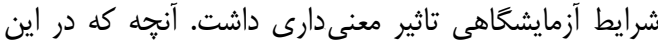

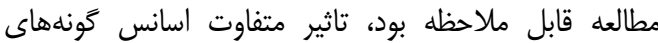

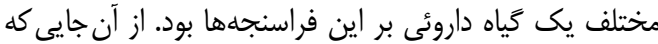

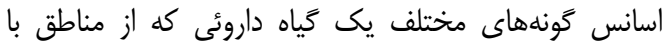

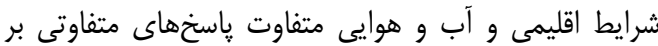

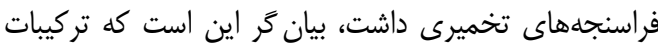

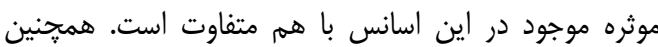

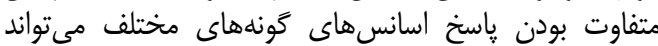

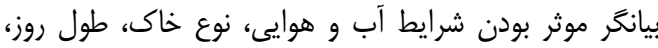

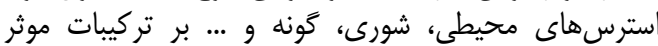

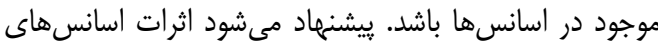

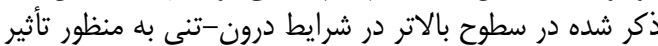

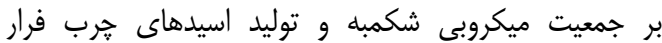

بررسى شود.

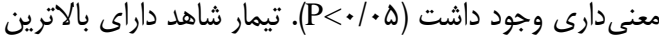

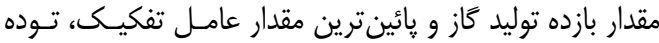

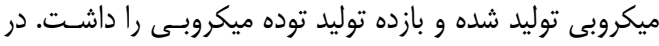

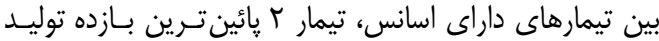

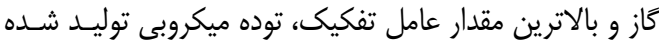

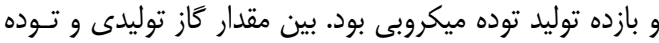

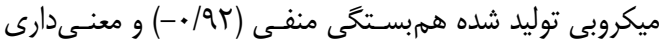

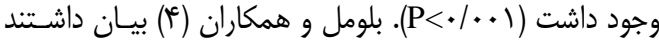

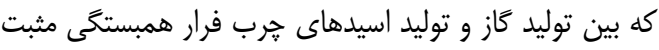

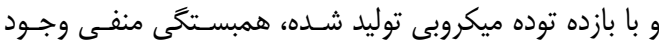

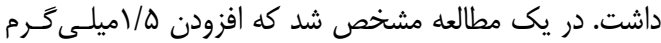

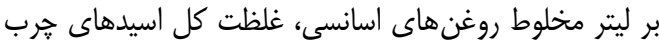

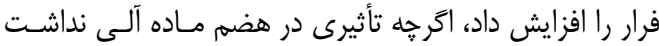

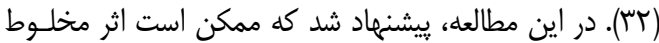
روغن هاى اسانسى مورد استفاده بر غلظت كل اسيدهاى

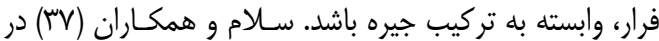

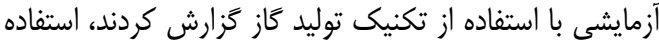

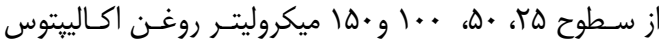

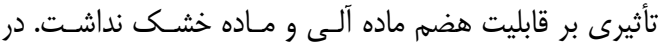

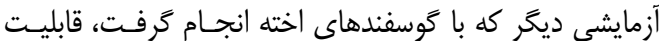

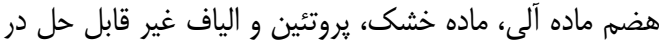

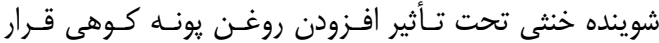

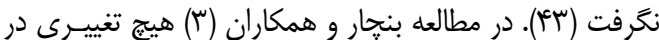

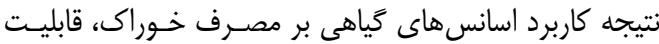

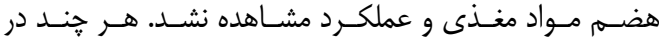

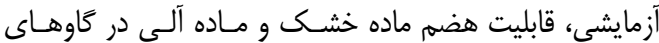

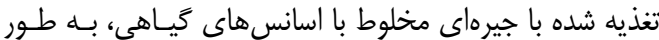

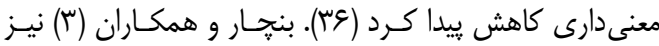

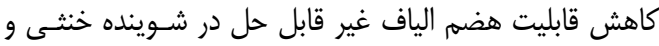

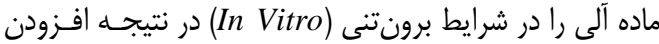

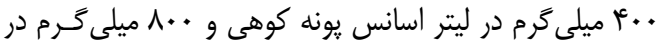

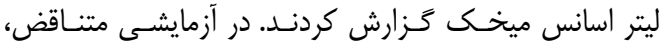

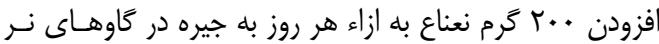

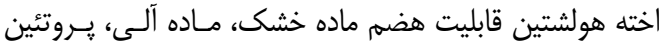

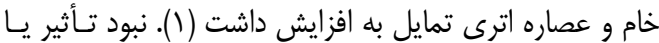

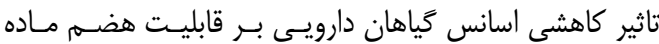

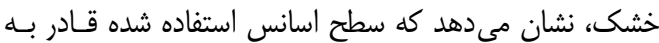

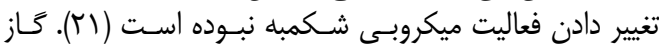

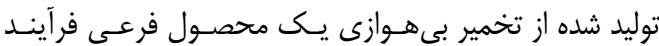

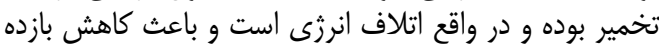

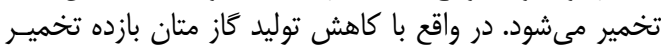
خوراك نيز افزايش قييدا مى كند. تُون

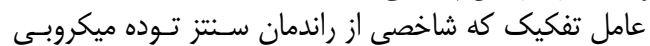

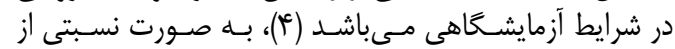


جدول r- تاثير اسانس گَونهاى مختلف كياه داروئى آويشن بر قابليت هضم ماده خشك و ماده آلى و فراسنجههاى تخميرى Table 3. Effect of various species of Thymus essence on digestibility, fermentation characteristics and microbial biomass production

\begin{tabular}{|c|c|c|c|c|c|c|c|}
\hline EMB & MB & $\mathrm{PF}$ & Gas yield $_{r \varphi}$ & $\mathrm{pH}$ & IVOMD & IVDMD & 'تيمارها' \\
\hline.$/ \Psi^{F^{c}}$ & $1 \% q / \backslash \Lambda^{D}$ & $r / \uparrow \Lambda^{c}$ & $r \Lambda T / T F^{\mathrm{a}}$ & $g / \mathrm{FV}^{\mathrm{a}}$ & $\mathrm{VQ} / \mathrm{qq}$ & $V \pi / T q$ & 1 \\
\hline$\cdot / 4 F \gamma^{a}$ & $1 \Delta T / V \cdot{ }^{a}$ & $r / \cdot r^{\mathrm{a}}$ & $\pi M F / T \varepsilon^{c}$ & $\varepsilon / \pi \omega^{c}$ & $V r / q \mu$ & $V M / . r$ & r \\
\hline . & $\mid F V / \Lambda r^{a}$ & $r / q$. a & $M F V / V \cdot{ }^{D C}$ & $s / \mathrm{H}^{\mathrm{Dc}}$ & $V \pi / r g$ & $v \cdot / \mu$. & r \\
\hline$. / F Y .{ }^{a b}$ & $\mid F T / \Delta \Lambda^{a b}$ & $r / \Lambda .^{D}$ & $r \Delta T / \Delta \delta^{\mathrm{DC}}$ & $\varepsilon / F^{\circ}{ }^{\circ}$ & $V \pi / \mu \Delta$ & $V . / . r$ & f \\
\hline$\cdot / 4 \cdot 9^{D}$ & $\mid r V / \cdot \wedge^{a b}$ & $r / V I^{D}$ & $r \Delta Q / \Lambda)^{D}$ & $s / \pi v^{\mathrm{Dc}}$ & $V r / q F$ & $99 / M$ & $\Delta$ \\
\hline 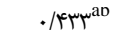 & $\mid \mp q / A r^{a}$ & $r / \Lambda q^{a b}$ & $M F \cdot / F D^{D}$ & $8 / K^{\circ}{ }^{\circ}$ & $V \Delta / .$. & $V r / r)$ & q \\
\hline$\% \Delta$ & $.1 \cdot 11$ & $\cdot / \cdot \Delta$ &.$/ \ldots r$ & $\cdot \cdots 1$ &.$/ 4 q$ & $\cdot / M F$ & $P$ Value \\
\hline .1 .11 & $0 / .9$ & .1199 & $1 r / 9 V$ & .1 .19 & או/ו & $1 / T V$ & SEM \\
\hline $\begin{array}{l}\text { Thymus s } n \\
\text { م به ازاء خـرم Gas yield } \\
\text { : }\end{array}$ & بى ترايط شبر & 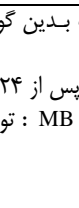 & 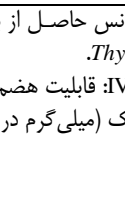 & 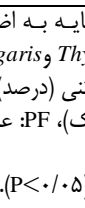 & 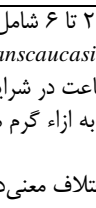 & 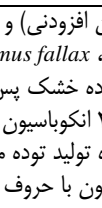 & 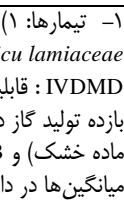 \\
\hline
\end{tabular}

1. Ando, S., T. Nishida, M. Ishida, K. Hosoda and E. Bayaru. 2003. Effect of peppermint feeding on the digestibility, ruminal fermentation and protozoa. Livestock Production Science, 82: 245-248.

2. Baghalian, K. and H. NaghdiBadi. 2000. Essenced plants. $1^{\text {st }}$ publication. Andarz publisher, P20-213103-104-105 (In persian).

3. Benchaar, C., V. Petit, R. HBerthiaume, D.R. Ouellet, J. Chiquette and P.Y. Chouinard. 2007. Effects of essential oils on digestion, ruminal fermentation, rumen microbial populations, milk production and milk composition in dairy cows fed alfalfa silage or corn silage. Journal of Dairy Science. 90: 886897.

4. Benchaar, C., T.A. McAllister and P.Y. Chouinard. 2008. Digestion, ruminal fermentation, ciliate protozoal populations and milk production from dairy cows fed Cinnamaldehyde, Quebracho Condensed Tannin, or Yucca schidigeraSaponin Extracts. Journal of Dairy Science, 91: 4765-4777.

5. Blummel, M., H.P.S. Makkar and K. Becker. 1997. In vitro gas production: a technique revisited. Journal of Animal Physiology and Animal Nutrition, 77: 34-24.

6. Borchers, R. 1965. Proteolytic activity of rumen fluid in vitro. Journal of Animal Science, 24: 1033 1038.

7. Calsamiglia, S., M. Busquet, P.W. Cardozo, L. Castillejos and A. Ferret. 2007. Invited Review: Essential oils as modifiers of rumen microbial fermentation. Journal of Dairy Science, 90: 2580-2595.

8. Carlton, R.R., A.I. Gray and P.G. Waterman. 1992. The antifungal activity of the leaf gland oil of sweet gale (Myrical gale). Chemecology, 3: 55-59.

9. Castillejos, L., S. Calsamiglia and A. Ferret. 2006. Effect of essential oil active compounds on rumen microbial fermentation and nutrient flow in in vitro systems. Journal of Dairy Science, 89: 2649-2658.

10. Castillejos, L., S. Calsamiglia, J. Martin-Tereso and H. Ter Wijlen. 2008. In vitro evaluation of effects of ten essential oils at three doses on ruminal fermentation of high concentrate feedlot-type diets. Animal Feed Science Technology, 145: 259-270.

11. Chaves, A.V., K. Stanford, E.R. Dugan, L.L. MGibson, T.A. McAllister, F. Van Herk and C. Benchaar. 2008. Effects of cinnamaldehyde, garlic and juniper berry essential oils on rumen fermentation, blood metabolites, growth performance, and carcass characteristics of growing lambs. Livestock Production Science, 117: 215-224.

12. Devant, M., A. Anglada and A. Bach. 2007. Effects of plant extract supplementation on rumen fermentation and metabolism in young Holstein bulls consuming high levels of concentrate. Animal Feed Science Technology, 137: 46-57.

13. Dorman, H.J.D. and S.G. Deans. 2000. Antimicrobial agents from plants: antibacterial activity of plant volatile oils. Journal of Applied Microbiology, 88: 308-316.

14. Dudareva, N., E. Pichersky and J. Gershenzon. 2004. Biochemistry of plant volatiles. Plant Physiology, 135: 1893-1902.

15. Duff, G.C., M.L. Galyean and M.E. Branine. 1995. Effects of adaptation to lasalocid,monensin or a daily rotation of lasalocid and mnensin on in vitro fermentation of a 90-percent concentrat diet. Journal of Animal Science, 75: 129-134.

16. Faleiro, M.L., M.G. Miguel, F. Ladeiro, R. Venancio, J.C. Tavares, A.C. Brito, J. Figueiredo, G. Barros and L.G. Pedro. 2003. Antimicrobial activity of essential oils isolated from Portuguese endemic species of Thymus. Letters in Applied Microbiology, 36: 35-40.

17. Garcia-Gonzalez, R., S. Lopez, M. Fernandez and J.S. Gonzalez. 2008. Dose-response effects of Rheum officinal root and Frangulaalnus bark on ruminal methane production in vitro. Animal Feed Science Technology, 145: 319-334.

18. Gershenzon, J., M.E. McConkey and R.B. Croteau. 2000. Regulation of monoterpene accumulation in leaves of peppermint. Plant Physiology, 122: 205-213.

19. Getachew, G., M. Blümmel, H.P.S. Makkar and K. Becker. 1998. In vitro gas measuring techniques for assessment of nutritional quality of feeds: A review. Animal Feed Science Technology, 72: 261281. 
20. Hristov, A.N., T.A. McAllister, F.H. Van Herk, K.J. Cheng, C.J. Newbold and P.R. Cheeke. 1999. Effect of Yucca schidigera on ruminal fermentation and nutrient digestion in heifers. Journal of Animal Science, 77: 2554-2563.

21. Jahani-Azizabadi, H., M. Danesh Mesgaran, A.R. Vakili, K. Rezayazdi and M. Hashemi. 2011. Effect of various medicinal plant essential oils obtained from semi-arid climate on rumen fermentation characteristics of a high forage diet using in vitro batch culture. African Journal of Microbiology Research, 5: 4812-4819.

22. James, T.K., A. Rahman and J.A. Douglas. 1991. Control of weeds in five herb crops.Proc. $44^{\text {th }}$ N.Z. Weed and Pest Control Conf.: 116-120.

23. Leung, A.Y. and S. Foster, 1996. Encyclopedia of common natural ingredients used in foods, drugs and cosmetics. A Wiley Inter science Publication- John Wiley and Sons, Inc, $649 \mathrm{pp}$.

24. Makkar, H.P.S. 2005. In vitro gas methods for evaluation of feeds containing phytochemicals. Animal Feed Science Technology, 123: 291-302.

25. Martinez, S., J. Madrid, F. Hernandez, M.D. Megias, J.A. Sotomayor and M.J. Jordan. 2006. Effect of thyme essential oils (Thymus hyemalis and Thymus zygis) and monensin on in vitro ruminal degradation and volatile fatty acid production. Journal of Agricultural and Food Chemistry, 54: 65986602 .

26. Menke, K.H., L.A. Salewski, H. Steingass, D. Fritz and W. Schneider. 1979. The estimation of the digestibility and metabolisable energy content of ruminant feeding stuffs from the gas production when they are incubated with rumen liquor. The Journal of Agricultural Science, 93: 217-222.

27. Menke, K.H. and H. Steingass. 1988. Estimation of the energetic feed value obtained from chemical analysis and in vitro gas production using rumen fluid. Animal research and development, 28: 7-55.

28. McGimpsey, J.A., M.H. Douglas, J.W. Van Klink, D.A. Beauregard and N.B. Perry. 1994. Seasonal variation in essential oil yield and composition from naturalized Thymus Vulgaris L. in Newzeeland Flavor and Fragrance Journal, 347-352.

29. Mohmeni, T.K.H. and N. Sharokhi. 1991. Essnces of plant and their remidation effects.Tehran University publication (In Persian).

30. Morton, J.F. 1977. Major medicinal plants, botany, culture and uses. Charles, C. Thomas Publisher, Bannerstone House, $431 \mathrm{pp}$.

31. Nagy, J.G. and R.P. Tengerey. 1968. Antibactrial action of essential oil of Artemisia as an ecological factor. II. Antibactrial action of volatel oil of Artemisia tiridentata (bigsagibrush) on buctria from the rumen of mule deer. Journal of Applied Microbiology, 16: 441-444.

32. Newbold, C.J., F.M. McIntosh, P. Williams, R. Losa and R.J. Wallace. 2004. Effects of a specific blend of essential oil compounds on rumen fermentation. Animal Feed Science Technology, 114: 105112.

33. O'Hara, M. and K. Ohki. 1973. Studies of the mode of gas production in an artificial rumen and its application to the evaluation of feedstuffs. III. The mode of volatile fatty acid production, and its relation to the gas production rate. Japanese Journal of Zootechnical science, 44: 432-439.

34. Oliveira, J.S., J.T. Huber, J.M. Simas, C.B. Theurer and R.S. Swingle. 1995. Effect of sorghum grain processing on site and extent of digestion of starch in lactating dairy cows. Journal of Dairy Science, 78: 1318-1327.

35. Ørskov, E.R. and I. McDonald. 1979. The estimation of protein degradability in the rumen from incubation measurements Weighed according to the rate of passage. The Journal of Agricultural Science, 92: 499-503.

36. Patra, A.K., D.N. Kamra and N. Agarwal. 2006. Effect of plant extracts on in vitro methanogenesis, enzyme activities and fermentation of feed in rumen liquor of buffalo. Animal Feed Science Technology, 128: 276-291

37. Sallam, S.M.A., I.C.S. Bueno, P. Brigide, P.B. Godoy, D.M.S. Vitti and A.L. Abdalla. 2009. Efficacy of eucalyptus oil on in vitro ruminal fermentation and methane production. Options Méditerranéennes, 85: 267- 272.

38. Skandamis, P.N. and G.J. Nychas. 2000. Development and evaluation of a model predicting the survival of Escherichia coli O157: H7 NCTC 12900 in homemade eggplant salad at various temperatures, pHs and oregano essential oil concentrations. Applied and Environmental Microbiology, 66: 1646-1653.

39. Talebzadeh, R., D. Alipour, M.J. Saharkhiz, A. Azarfar and M. Malecky. 2012. Effect of essential oils of Zataria multiflora on in vitro rumen fermentation, protozoal population, growth and enzyme activity of anaerobic fungus isolated from Mehraban sheep. Animal Feed Science Technology, 172: 115-124.

40. Theodorou, M.K., B.A. Williams, M.S. Dhanoa, A.B. McAllan and J. France. 1994. A simple gas production method using a pressure transducer to determine the fermentation kinetics of ruminant feeds. Animal Feed Science Technology, 48: 185-197.

41. Van Soest, P.J. 1994. Nutritional Ecology of the Ruminant. Cornel University Press, Ithaca, New York, $374 \mathrm{pp}$.

42. Wang, Y., T.A. McAllister, C.J. Newbold, L.M. Rode, P.R. Cheeke and K.J. Cheng. 1998. Effects of Yucca schidigera extract on fermentation and degradation of steroidal saponins in the rumen simulation technique (RUSITEC). Animal Feed Science Technology, 74: 143-153.

43. Wang, C.J., S.P. Wang and H. Zhou. 2009. Influences of flavomycin, ropadiar and saponin on nutrient digestibility, rumen fermentation and methane emission from sheep. Animal Feed Science Technology, 148: 157-166. 


\title{
The Effects of Various Species of Thymus Essence on Gas Production Parameters, Dry Matter and Organic Matter Digestibility and Ruminal Fermentation Parameters in In Vitro
}

\author{
Maryam Fereydoonpoor ${ }^{1}$, Javad Bayatkouhsar ${ }_{4}^{2}$ Ebrahim Gholamali Pour ${ }^{3}$ and \\ Poneh Ebrahimi
}

1 and 3- Gradated M.Sc. and Assistant Professor, University of Gonbab Kavus

2- Assistant Professor, University of Gonbab Kavus (Corresponding author: javad_bayat@yahoo.com) 4- Assistant Professor, Golestan University

Received: June 3, $2014 \quad$ Accepted: June 9, 2015

\begin{abstract}
Two experiments were conducted to evaluate the effect of essence of various species of Thymus on in vitro gas production parameters and digestibility of dry matter and organic matter. Various species of Thymus medicinal plant was collected from different area: Shriaz (Zataria multiflora), Esfarayen (Thymus transcaspicus lamiaceae), Shahrood (Thymus fallax), Golestan forestry (Thymus transcauasicus) and Gorgan (Thymus vulgaris). Essence of collected samples was taken by macro wave at $110{ }^{\circ} \mathrm{C}$ and $450 \mathrm{~nm}$ for 45 minutes. Treatments were including: 1) control containing basal diet with forage: concentrate ratio 50:50 (without essence) and treatment 2 till 6 containing basal diet and essence of various species. Results showed that adding essences from various species had significant effects on potential gas production and gas production rate $(\mathrm{p}<0.05)$ as treatment of control had highest potential gas production $(396.6 \mathrm{ml}$ per g dry matter) compared to other treatments. It was also observed that there were significant differences among essence containing treatments compared with control treatment on estimated parameters $(\mathrm{p}<0.05)$. The use of essence caused significant decrease in $\mathrm{pH}$ of culture medium compared to control treatment $(\mathrm{p}<0.05)$. In this experiment, there were no significant differences among various treatments in DM and OM digestibility $(\mathrm{p}<0.05)$. However, treatments that containing essence had higher microbial biomass production and microbial biomass production efficiency. It can be concluded that essence of different thymus varieties has the potential to affect ruminal fermentation efficiency and further researches is needed to determine the optimal doses of different thymus varieties essence.
\end{abstract}

Keywords: Digestibility, Essence, In vitro, Rumen fermentation 